\title{
The application of magnetic fluids in sealing nodes designed for operation in difficult conditions and in machines used in sea environment
}

\author{
Leszek Matuszewski, Ph.D. \\ Gdansk University of Technology \\ Zbigniew Szydło, Ph.D. \\ AGH University of Science and Technology
}

\author{
ABSTRACT
}

\begin{abstract}
Presented in article MF seals are being researched for sea technology purposes due to their excellent tightness and low resistance of motion. These features are most valuable for ring propellers and ship's main propeller shaft. There are more reasons why technologists payf significant attention for various $M F$ seals applications in their difficult operating conditions. For instance, an advanced pumping systems are designed with contactless (screw or centrifugal) fluid seal used as the first stage seal and then the MF seal as the main seal. Further parts of the article contain discuss of the properties of magnetic fluids in the construction of MF seals. Sample of the few systems of magnetic fluid seals are presented, which have been researched lately in our laboratory including sea conditions. The magnetic fluids used in our seals are colloidal suspensions of magnetic nanoparticles. Undertaken earlier various systems selection for sea-water purposes forced us to focus on double sealing systems, in which the MF seal is used as the second seal following a conventional system especially adapted to co-operation with MF systems. This solution successfully limits direct contact of the sealed liquid with the magnetic fluid, and the entire sealing construction secures absolute tightness of the system. Using this solution we also can reduce costs by elements high accuracy avoidance. Research and design activities are carried out in the AGH Laboratory of Seals and Magnetic Fluid Applications together with Deep Water Department of Technical University of Gdansk over the use of magnetic fluid seals in sea water environment. These activities are oriented on working out MF seals able to work effectively in machines in which low-pressure water flow takes place. The objects of experimental investigations are seals having two different nominal diameters: $50 \mathrm{~mm}$ and $220 \mathrm{~mm}$ and

two research rigs of different construction had to be built up. Works are scheduled to be continued.
\end{abstract}

Keywords: FM seal, sealing, magnetic fluids, sea technology, propellers

\section{INTRODUCTION}

Sealing rotating shafts, bearings, and other mechanical systems with the aid of the magnetic fluid, which creates a sealing partition kept steady by magnetic forces, is the technique which has been successfully used in machines working in gas environment at pressures up to, approximately, 1.0 MPa. These seals also provide opportunities for solving sealing problems in fixed and moving passages in vacuum machines.

In the worldwide technical literature and publications issued by companies producing magnetic fluids and machines in which they are applied the following names are used for the magnetic fluid seals:

+ FerroMagnetic Seal (acronym: FM Seal)

+ Magnetic Fluid Seal (acronym: MF Seal)

+ FerroFluidic Seal (acronym: FF Seal).

In further parts of the article the names: "Magnetic Fluid Seal" or "MF Seal" will be most frequently used.

As a result of numerous experimental and theoretical studies oriented on investigating distributions of the magnetic field in the seals and rheological properties of the magnetic fluids, complemented by the development of technologies for production of these fluids, certain principles have been formulated on magneto-mechanical shaping of the MF seal, selecting the type of fluid appropriate for expected conditions of machine operation, and calculating geometrical parameters of the sealing lips $[1,2,3,4]$.

Since the mechanism that keeps the MF seal tight differs considerably from that observed in other types of seals, opinions can be found that the use of MF seals is limited mainly to precise machines. These opinions are claimed to be justified by specific properties of magnetic fluids, the necessity of use of high-energy permanent magnets, and relatively high precision required during production of the MF seals. These factors decrease the permissible pressure limit to about $1.0 \mathrm{MPa}$, at the corresponding critical temperature of the magnetic fluid not exceeding, approximately, $200^{\circ} \mathrm{C}$. Moreover, extremely high prices of high-dispersion magnetic fluids (nanomagnetic fluids) and the abovementioned required precision of machining are the reasons why MF seals are not in common use at present.

However, despite the above difficulties, obvious advantages of these seals, including excellent tightness and low resistance of motion, are the reasons why growing interest is observed towards their use in difficult operating conditions, where they are mainly used as so-called protective seals. This refers to such machines as reactors used in the chemical, biochemical, 
and pharmaceutical industry, machines and systems used in the refining industry, and driving systems working in partial or total immersion in a liquid. Those machines frequently work in difficult conditions, which include non-uniformity of motion, oscillations and/or vibrations, high temperature and the action of aggressive environment having the form of vapours and aerosols. In the case of machines like floodable pump drives or sea-going vessel driving systems, which are immersed in liquids, certain problems connected with the contact of the magnetic fluid with the water are to be solved. The use of MF seals in those conditions requires special constructional designs and special magnetic fluids, which is of particular importance for machines used in liquids environment.

It is well known from available publications and patent solutions that the magnetic fluids applied in those conditions should reveal strong hydrophobic properties - here the most often described designs are hybrid sealing systems, in which the MF seal is used as the second seal following a conventional seal in a row arrangement. This design limits direct contact of the sealed liquid with the magnetic fluid, while the MF seal secures absolute tightness of the entire system.

For instance, some rotor pump sealing systems are designed in the above way, with the front seal or contactless (screw or centrifugal) fluid seal used as the first seal and the MF seal as the second seal.

In the case of high-amplitude vibrations, elastic systems are used which provide opportunities for stabilisation of the seal within certain range of its positions, despite the presence of oscillations of the sealed shaft. When high temperatures of operation are expected, the applied magnetic fluids should reveal increased temperature resistance, and intensive cooling of the system of magnets and the magnetic fluid is to be provided.

The next parts of the article discuss the properties of magnetic fluids, along with basic problems in the construction of MF seals. Sample designs of magnetic fluid seals are presented, which have been used in objects operating in difficult conditions, such as those observed, for instance, on sea-going vessels.

\section{MAGNETIC AND MAGNETORHEOLOGICAL FLUIDS}

The magnetic fluids used in seals are colloidal suspensions of magnetic nanoparticles (for instance magnetite $-\mathrm{Fe}_{2} \mathrm{O}_{3}$ ), of about $10 \mathrm{~nm}$ in diameter, in the carrier liquid (for instance, synthetic oil, silicone liquid, water). Particles having those dimensions are kept in constant motion by internal energy of the carrier liquid and create stable structures. In the production process, a surface active substance (oleic acid, for instance) is introduced to the carrier liquid to cover the surfaces of the magnetic particles and thus protect against their aggregation and sedimentation of the created multi-particle magnetic aggregates under the influence of gravitation and magnetic forces.

Magnetic fluids revealing those properties bear the name of nanomagnetic fluids. A basic advantage of the nanomagnetic fluids is high stability and durability of the colloidal system - which for many liquids can last as long as about 10 years.

Using a similar procedure to the described above, magnetic fluids are produced in with the suspensions of magnetic microparticles (magnetite $-\mathrm{Fe}_{2} \mathrm{O}_{3}$ ) have from 0.5 do $8 \mu \mathrm{m}$ in diameter. Their percentage content in the liquid is from $20 \%$ to $80 \%$ [6].

To make distinction between them and the nanomagnetic fluids, these liquids are called micromagnetic fluids, although in producers' catalogues and the professional literature they are more often referred to as magnetorheological fluids.

The micromagnetic fluids are not stable - when the magnetic particles remain in rest from several to several hundred hours, they are subject to sedimentation. However, if the energy from outside is delivered to this liquid, in the form of vibrations of motion of a mechanical system for instance, then the magnetic microparticles compose a homogeneous suspension, the behaviour of which is similar to that presented by the colloidal suspension of nanomagnetic particles. The main advantage of the micromagnetic fluids is high range of magnetic saturation - many times higher than that for nanomagnetic fluids, which increase their resistance to the action of external agents. Another important property is their rheological characteristic: strong and repeatable relation between the viscosity and the magnetic field strength - hence the frequently used name of a "magnetorheological fluid".

With respect to magnetic interactions, the magnetic (nanomagnetic and micromagnetic) fluids belong to the group of so-called superparamagnetic materials, i.e. materials which do not reveal, in practice, any magnetic hysteresis.
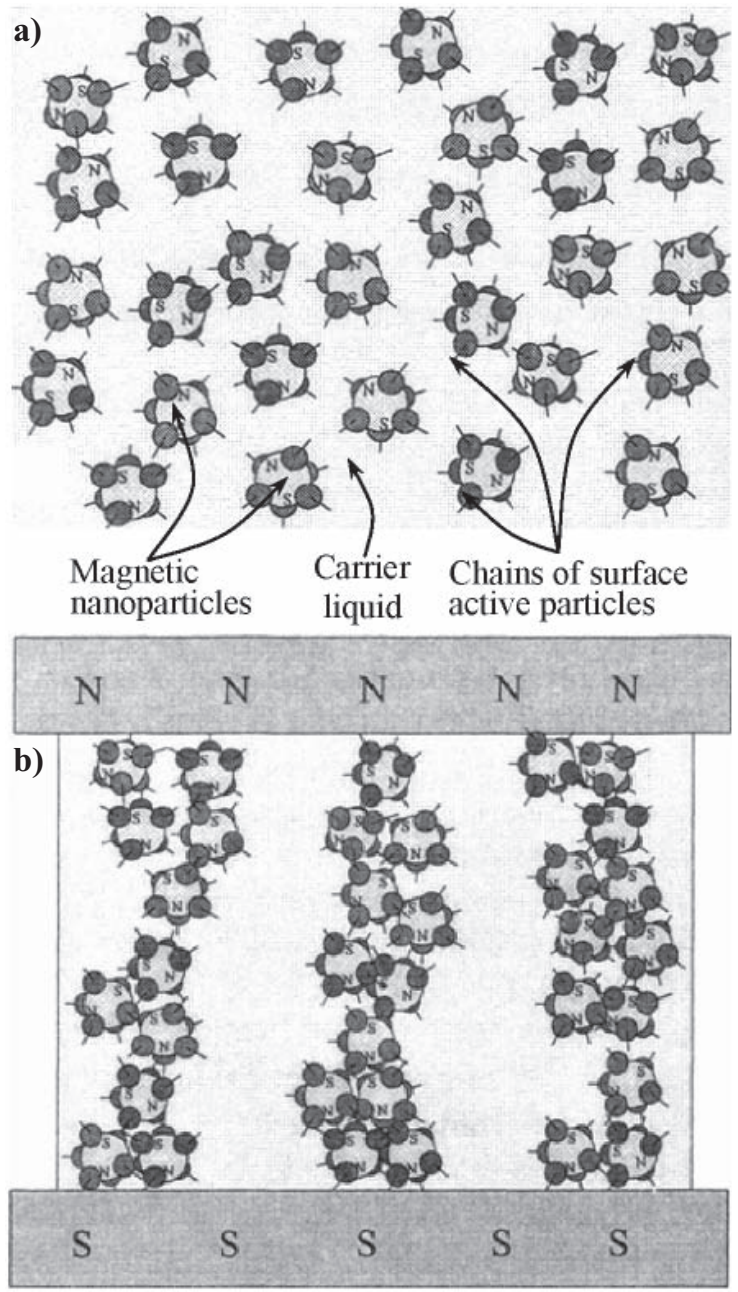

Fig. 1. Structure of the magnetic fluid and distribution of particles magnetic dipoles: a) disordered distribution of magnetic dipoles in the absence of magnetic field, $\boldsymbol{b})$ ordered chains of particles with oriented

directions of magnetic dipoles in the presence of magnetic field [6].

Fig. 1a shows the disordered structure of the magnetic fluid in the absence of the magnetic field. The particles compose magnetic dipoles of arbitrary orientation, which makes the resultant magnetic force equal zero.

Fig. 1b, in turn, shows the ordered structure of the magnetic fluid in the presence of a constant or varying magnetic field. In these circumstances the particles compose chains of magnetic dipoles oriented along the direction of the magnetic lines of force.

High flexibility and speed of response (from $0,01 \mathrm{~s}$ to $0,0001 \mathrm{~s}$ ) of the magnetic fluid to the presence of the magnetic field make the process of making order in particle orientations very short. 
This property provides opportunities for using magnetic fluids not only in seals and lubricating technology, but also in controlled vibration dampers and couplings, and in measuring instruments. Due to remarkable advancement in technology of colloids, the magnetic fluids can be manufactured based on carrier liquids adapted to the planned type of application and conditions of magnetic fluid operation, with various content and properties of the magnetic phase (nanoparticles or microparticles). All this provides good opportunities for controlling parameters of the magnetic fluid and adapting them to actual needs.

Basic physical properties of the magnetic fluids, which decide on their possible applications, include magnetic saturation, dynamic viscosity, and operating temperature range. The magnetic saturation of the liquid determines the permissible pressure level (critical pressure) of the seal, while the viscosity affects the resistance of motion, and the range of temperatures in which the magnetic fluid can be used is to be adapted to operating conditions of a given machine.

At present, magnetic fluids are produced which reveal magnetic saturation up to $70 \mathrm{kA} / \mathrm{m}$ and dynamic viscosity ranging from 2 to $15000 \mathrm{mP} \cdot \mathrm{s}$.

The micromagnetic fluids reveal much higher, from five to ten times, magnetic saturation, which is connected with much larger dimensions of the magnetic particles used in those liquids.

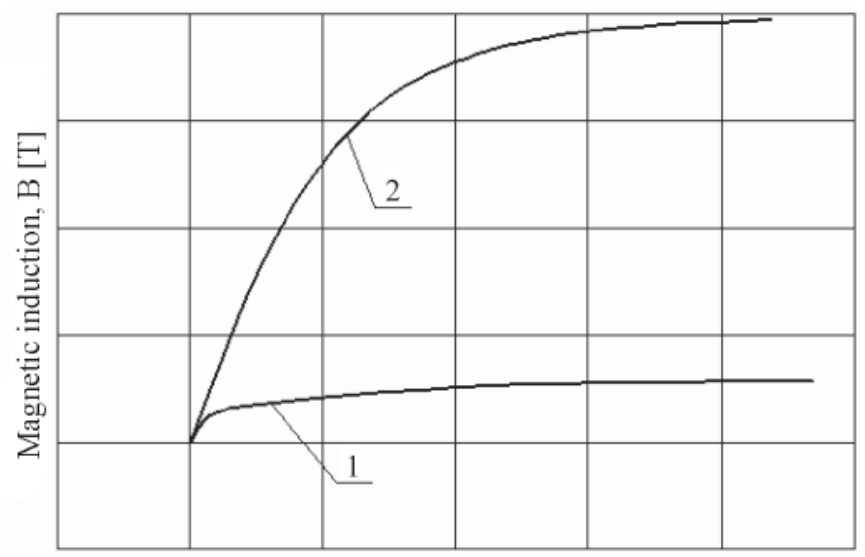

Strength of magnetic field, $\mathrm{H}[\mathrm{kA} / \mathrm{m}]$

Fig. 2. Magnetic induction vs. magnetic field strength: nanomagnetic fluid (1) and magnetorheological fluid (2)

Fig. 2 shows the dependence of the magnetic induction B in the nanomagnetic and micromagnetic (magnetorheological) liquids on the strength $\mathrm{H}$ of the external magnetic field [6].

Tab. 1 collects basic properties of the nanomagnetic fluids produced by FerroLabs, Russia, while in Tab. 2 basic properties of the magnetorheological (micromagnetic) fluids produced by

Tab. 1. Properties and applications of magnetic (nanomagnetic) fluids produced by FerroLabs, Russia, and used in sealing and lubricating technology

\begin{tabular}{|c|c|c|c|c|c|c|}
\hline $\begin{array}{c}\text { Type of } \\
\text { fluid }\end{array}$ & $\begin{array}{l}\text { Carrier } \\
\text { liquid }\end{array}$ & $\begin{array}{c}\text { Operating } \\
\text { temperature } \\
\text { range. }{ }^{\circ} \mathrm{C} \\
\end{array}$ & $\begin{array}{c}\text { Density } \\
\text { g/cm }\end{array}$ & $\begin{array}{c}\text { Viscosity } \\
\text { Pa.s } \\
\text { a } 25^{\circ} \mathrm{C}\end{array}$ & $\begin{array}{l}\text { Magnetic } \\
\text { saturation. } \\
\mathbf{k A} / \mathbf{m}\end{array}$ & Applications \\
\hline $\begin{array}{c}\text { FLS } \\
300.020\end{array}$ & $\begin{array}{l}\text { Silicone } \\
\text { liquid }\end{array}$ & $-50 \ldots+90$ & $1.2 \ldots 1.4$ & $0.5 \ldots 0.8$ & $20 \ldots 30$ & $\begin{array}{l}\text { Vacuum machines: } \\
\text { - plasma coating } \\
\text { - semiconductor technology devices }\end{array}$ \\
\hline $\begin{array}{c}\text { FLS } \\
040.040\end{array}$ & $\begin{array}{l}\text { Silicone } \\
\text { liquid }\end{array}$ & $-70 \ldots+150$ & $1.0 \ldots 2.0$ & $0.3 \ldots 0.8$ & $40 \ldots 50$ & $\begin{array}{l}\text { High-speed shafts: } \\
\text { - chemical reactors } \\
\text { - CD and DVD drives }\end{array}$ \\
\hline $\begin{array}{c}\text { FLS } \\
250.020\end{array}$ & $\begin{array}{l}\text { Silicone } \\
\text { liquid }\end{array}$ & $-60 \ldots+100$ & $1.1 \ldots 2.2$ & $\ldots .1 .0$ & $20 \ldots 30$ & $\begin{array}{l}\text { Dustproof seals: } \\
\text { - precise devices } \\
\text { - measuring instruments }\end{array}$ \\
\hline $\begin{array}{c}\text { FLF } \\
750.030\end{array}$ & $\begin{array}{l}\text { Perfluoro- } \\
\text { polyether }\end{array}$ & $-30 \ldots+130$ & 2.05 & $\ldots .15$ & $30 \ldots 40$ & $\begin{array}{l}\text { Seals against aggressive gases and liquids: } \\
\text { - industrial chemical reactors } \\
\text { - petrochemical facilities }\end{array}$ \\
\hline $\begin{array}{c}\text { FLW } \\
250.020\end{array}$ & Water & $0 \ldots+90$ & 1.2 & .....0.02 & $5 \ldots 30$ & $\begin{array}{l}\text { Facilities in biotechnology and medicine: } \\
\text { - medical instruments } \\
\text { - production machines }\end{array}$ \\
\hline $\begin{array}{c}\text { FLC } \\
002.050\end{array}$ & Kerosene & $-50 \ldots+50$ & 1.2 & $\begin{array}{c}0.002 \ldots \\
0.02\end{array}$ & $30 \ldots 70$ & $\begin{array}{l}\text { Magnetic sensors and separators: } \\
\text { - position and acceleration sensors } \\
\text { - separation of materials }\end{array}$ \\
\hline $\begin{array}{c}\text { FLC } \\
050.025\end{array}$ & $\begin{array}{c}\text { Synthetic } \\
\text { oil }\end{array}$ & $-50 \ldots+150$ & $1.0 \ldots 2.0$ & $\ldots .0 .3$ & $25 \ldots . .50$ & $\begin{array}{l}\text { Lubricating magnetic fluid: } \\
\text { - hermetic bearing units lubricated with magnetic fluid }\end{array}$ \\
\hline
\end{tabular}

Tab. 2. Properties and applications of magnetorheological (micromagnetic) liquids produced by FerroLabs, Russia, and Lord, USA, and used in controlled vibration dampers, couplings, brakes, and in abrasive machining of materials

\begin{tabular}{|c|c|c|c|c|c|c|}
\hline Type of fluid & $\begin{array}{c}\text { Carrier } \\
\text { liquid }\end{array}$ & $\begin{array}{c}\text { Operating } \\
\text { temperature } \\
\text { range. }{ }^{\circ} \mathbf{C}\end{array}$ & $\begin{array}{c}\text { Density } \\
\mathbf{g} / \mathbf{c m}^{\mathbf{3}}\end{array}$ & $\begin{array}{c}\text { Viscosity } \\
\mathbf{P a} \mathbf{s} \\
\mathbf{\mathbf { 2 }} \mathbf{2 5}^{\circ} \mathbf{C}\end{array}$ & $\begin{array}{c}\text { Magnetic } \\
\mathbf{s a t u r a t i o n} \\
\mathbf{k A} / \mathbf{m}\end{array}$ & Applications \\
\hline $\begin{array}{c}\text { FLS 040.600/ } \\
\text { FerroLabs }\end{array}$ & $\begin{array}{c}\text { Silicone } \\
\text { liquid }\end{array}$ & $-50 \ldots+150$ & 3.28 & $\ldots .10$ & $600 \ldots 700$ & $\begin{array}{c}\text { Controlled brakes, couplings, dampers, } \\
\text { vibration insulators }\end{array}$ \\
\hline $\begin{array}{c}\text { FLW 001.300/ } \\
\text { FerroLabs }\end{array}$ & Water & $0 \ldots+90$ & 3.42 & $\ldots .10$ & $300 \ldots 600$ & Magnetic abrasive machining of materials \\
\hline $\begin{array}{c}\text { MRF-132ED/ } \\
\text { Lord }\end{array}$ & $\begin{array}{c}\text { Synthetic } \\
\text { oil }\end{array}$ & $-40 \ldots+150$ & 3.06 & $\begin{array}{c}0.33 \\
\ldots .0 .94\end{array}$ & - & $\begin{array}{c}\text { Controlled brakes, couplings, dampers, } \\
\text { vibration insulators }\end{array}$ \\
\hline $\begin{array}{c}\text { MRF-240BS/ } \\
\text { Lord }\end{array}$ & Water & $0 \ldots+70$ & 3.82 & $\begin{array}{c}5.0 \\
\ldots .13 .6\end{array}$ & - & $\begin{array}{c}\text { Educational purposes. demonstration } \\
\text { of magnetorheological effects }\end{array}$ \\
\hline
\end{tabular}


FerroLabs and Lord, USA, are given. According to FerroLabs, the magnetic saturation of the magnetorheological fluids reaches up to $700 \mathrm{kA} / \mathrm{m}$. The American company Lord does not publish the level of magnetic saturation of the magnetorheological liquids produced by them.

\section{THE STRUCTURE AND PRINCIPLE OF OPERATION OF MAGNETIC FLUID SEALS}

Of particular importance among technical applications of the magnetic fluids are MF seals for rotating shafts. The magnetic fluid seals (MF seals) compose a separate class of contactless seals, revealing absolute tightness (leakage at the level of $10^{-6} \mathrm{~Pa} \cdot 1 / \mathrm{s}$ ) within the range from very high vacuum $\left(10^{-6} \mathrm{~Pa}\right)$ do the pressures of an order of $1.0 \mathrm{MPa}$, at relatively small power losses generated by liquid friction. For the time being, the MF seals have been used mainly in gas environment: as protective seals for rolling bearings, seals for passages of rotating shafts in vacuum technology machines, and as shaft seals in fans, mixers, and chemical and biochemical reactors.

Fig. 3 shows the structure and principle of operation of the rotating shaft MF seal. A permanent magnet is fixed in the casing made of non-magnetic material. The magnet has the shape of a cylinder with an opening. The NS magnetisation direction of the cylinder is parallel to the axis of the sealed shaft. One cylindrical pole shoe made of magnetic material is situated on each side of the permanent magnet. Between the shaft made of magnetic material and the inner surface of the pole shoe openings there is a gap filled with the magnetic fluid. The fluid is kept in place by magnetic forces generated by the permanent magnet closed in the circuit composed by pole shoes, shaft and the magnetic fluid. The thickness of the gap with the magnetic fluid ranges, generally, between $0.1 \mathrm{~mm}$ and $0.3 \mathrm{~mm}$.

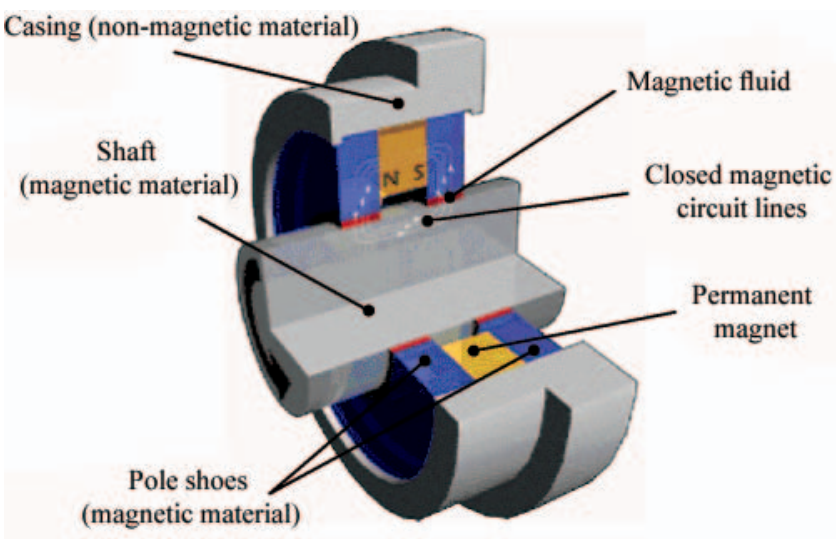

Fig. 3. Basic components of rotating shaft MF seal [7]

Despite simple principle of operation and construction of MF seals, designing them properly requires special knowledge due to specific properties of the magnetic fluid and its behaviour in the magnetic field. This refers in particular to largedimension rotating shafts in high-speed machines revealing complex kinetics of motion.

MF seal's ability to keep tightness is defined by a so-called limiting perforation pressure, which is the maximum pressure kept by the seal, above which the magnetic fluid is ejected out from the gap and the tightness is lost. The ability to keep the seal tight depends on magnetic saturation of the liquid, the level of filling the gap with the magnetic fluid, the geometry of the seal, and pressure difference between two sides of the seal.

Fig. 4 shows the volumetric deformation of the magnetic fluid in the magnetic field in the gap of a single sealing lip.
The deformation is generated by the pressure difference between areas 4 and 1 .

The pressure difference $\Delta \mathrm{p}$ which provokes the perforation of a single sealing lip filled with the magnetic fluid was determined from the Bernoulli equation for the flow of liquid in the magnetic field [2]. This equation takes into account only the magnetic saturation $\mathrm{M}_{\mathrm{s}}$ of the magnetic fluid and the difference between the maximum and minimum values, $\mathrm{H}_{\max }$ and $\mathrm{H}_{\min }$, of the magnetic field in the working gap.

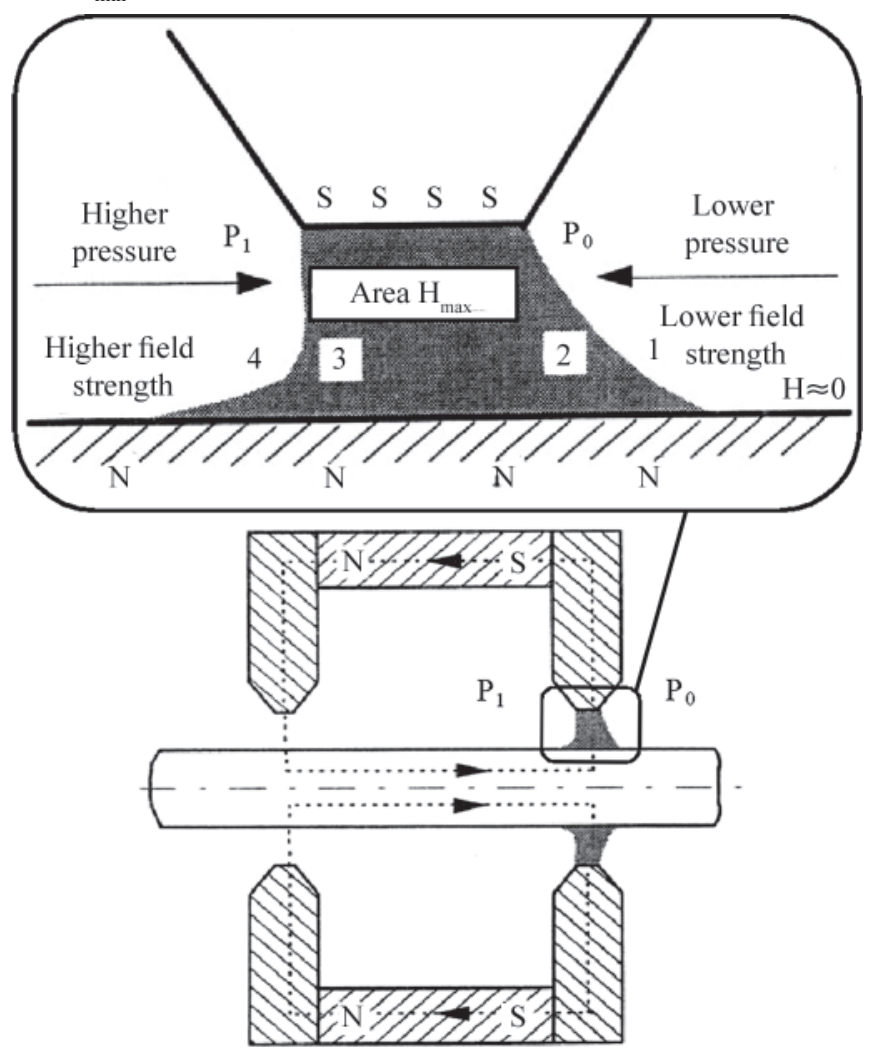

Fig. 4. Principle of operation of the magnetic fluid seal. 1 - lower pressure area, 2 - lower magnetic field strength area, 3 - higher magnetic field strength area, 4 - higher-pressure area

The distribution of the magnetic field (induction) within the gap, and consequently $\mathrm{H}_{\max }$ and $\mathrm{H}_{\min }$, depend on the geometry of the sealing lip, the height of the working gap, and the energy of the magnetic system.

After assuming $\mathrm{H}_{\min }=0$ we arrive at the relation which will allow us to determine $\Delta \mathrm{p}$ for a single sealing lip in static conditions $\left(\mu_{\mathrm{o}}-\right.$ magnetic permeability of the vacuum):

$$
\Delta \mathrm{p}=\mu_{\mathrm{o}} \mathrm{M}_{\mathrm{s}} \mathrm{H}_{\max }=\mathrm{M}_{\mathrm{s}} \mathrm{B}_{\max }
$$

Determining the critical pressure for the MF seal in dynamic conditions in much more difficult, as the magnetic fluid in the working gap is subject to the action of not only the magnetic forces, but also flow processes, rotation (spin) of particles, their mutual interactions, and the centrifugal force which affects the position of the magnetic fluid in the gap and changes the seal perforation pressure. This pressure depends on the position of the sealing lips, which are mounted either on the rotating shaft, or on the pole shoes steadily fixed in the casing. These phenomena are clearly visible at higher rotational speeds of the shaft - above $10 \mathrm{~m} / \mathrm{s}$.

The results of the experimental investigations suggest that the critical perforation pressure for a single sealing lip in the arrangement shown in Fig. 3 is approximately equal to $0.05 \mathrm{MPa}$. A similar pressure level per one sealing lip can be assumed for a symmetrical two-lip arrangement, which is 
more rational than a single lip from the technical point of view. However, for multi-lip seals (in symmetrical arrangements with four, six and more lips) the permissible working pressure for a single sealing lip is to be assumed from within the range between $0.03 \mathrm{MPa}$ and $0.04 \mathrm{MPa}$.

\section{MAGNETIC FLUID SEALS FOR ROTATING SHAFTS WITH RADIAL AND AXIAL RUNOUT}

Using magnetic fluid seals for sealing rotating shafts with axial and radial runout requires special designs. When the axial and/or radial runout is relatively small, the applied sealing constructions are flexibly mounted on the machine casing using elastic rings. In the case of large axial and radial runout, the sealing constructions are mounted on elastic bellows fixed to the machine casing.

Fig. 5 shows the construction of a sealing passage with the magnetic fluid used in chemical reactors, in which extremely high tightness of the process chamber is required [7].

The sealing unit consists of a permanent magnet 5 with axial polarisation, the pole shoes 1 , and a sleeve 11 with sealing lips, fixed to the bearing casing 3 on surface 15 . The sleeve 11 is made of magnetic material, while the casing 3 is made of nonmagnetic material. As a result, the magnetic circuit is closed through the magnetic fluid 10 . The pole shoes 1 are placed inside the sleeve 4 , which is rotationally mounted in the casing 3 using the rolling bearings 2 . The sleeve 4 is connected with the shaft 8 via a moving sleeve 6 . Loose connections between the shaft 8 , the sleeve 6 , and the sleeve 4 , along with the application of elastic sealing O-rings 7 and 9 in those connections, provide opportunities for good transmission of vibrations, at the same time keeping the thickness of the gap 10 with the magnetic fluid constant. Loose fixing of the casing 3 in the flange 13, done with the aid of the sealing O-ring 12, also makes it possible to transmit axial displacements of the shaft.

This construction makes it possible to keep the seal tight even when drive shaft vibrations reach as much as $0.7 \mathrm{~mm}$.

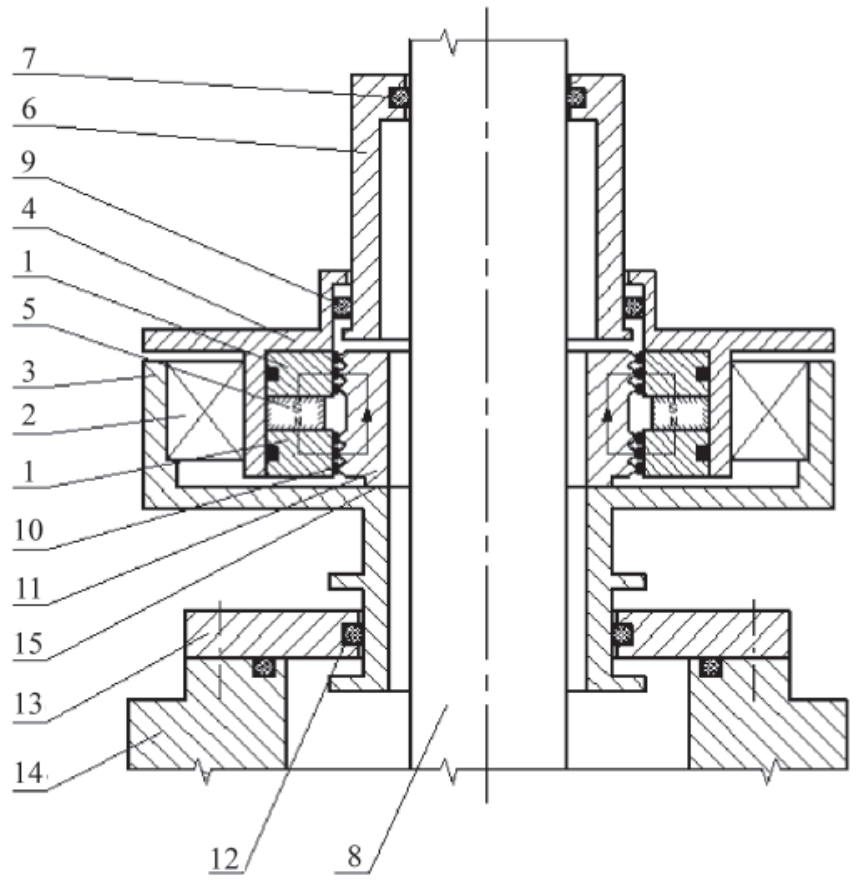

Fig. 5. Magnetic fluid seal for vertical shaft with limited axial runout: $\mathbf{1}$-pole shoe; $\mathbf{2}$-thrust bearing; $\mathbf{3}$ - bearing casing; $\mathbf{4}$-intermediate sleeve; 5 - permanent magnet; $\mathbf{6}$ - moving sleeve; 7, 9, 12 - sealing O-rings; $\boldsymbol{8}$-shaft; 10 - magnetic fluid; $\mathbf{1 1}$-sleeve with sealing lips; 13 - flange; 14 - casing; 15 -fixing of sleeve 11 with sleeve 3.
Fig. 6 shows the magnetic fluid seal for a shaft with small axial and radial runout [9]. This seals consists of an axially polarised permanent ring magnet 1 , pole shoes 2,3 mounted on the shaft 5 , and two self-adjusting rings 9,10 . The shaft 5 is made of non-magnetic material. The casing 8 , the permanent magnet 1 with the pole shoes 2,3 , the self-adjusting rings 9,10 and the magnetic fluid 12 filling the radial gaps $\delta_{1}, \delta_{2}$ and the axial gaps $\delta_{3}, \delta_{4}$ compose a closed magnetic circuit with magnetic flux $\Phi$. The magnetic flux generates a radial force, which moves the self-adjusting rings 9,10 closer to the casing 8 , and a radial force which presses the self-adjusting rings, via rolling elements 11 , to the pole shoes 2,3 . The magnetic force keeps the magnetic fluid 12 in the radial and axial gaps, thus securing tightness of the entire sealing structure.

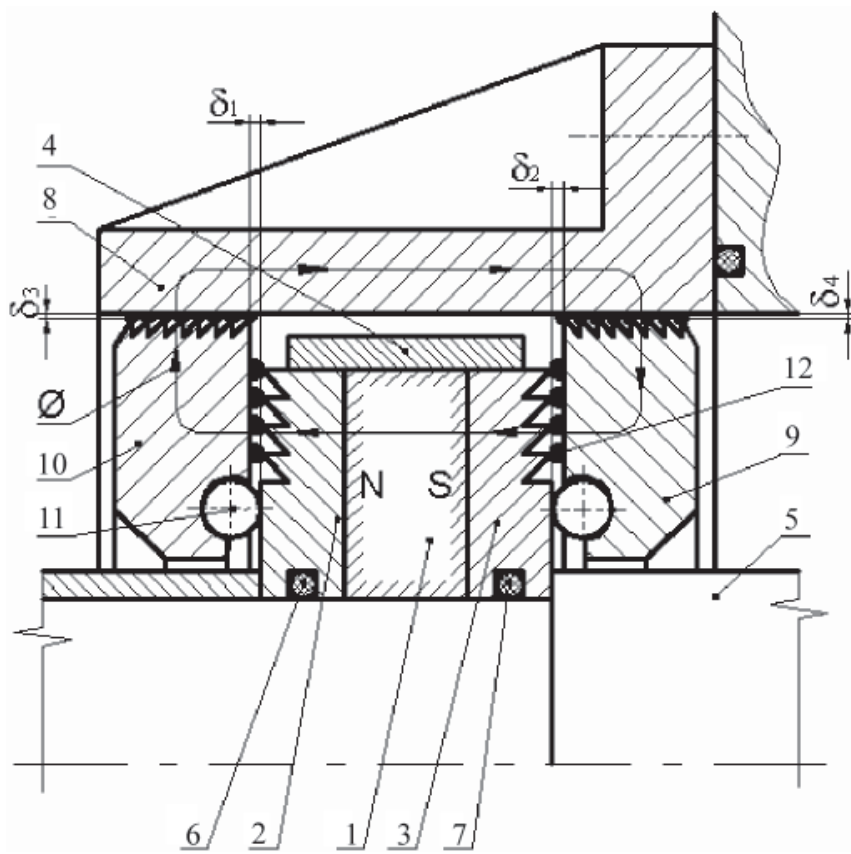

Fig. 6. Magnetic fluid seal for a shaft with limited axial and radial runout $\mathbf{1}$-permanent magnet; 2,3 -pole shoes; 4 - clamping ring; 5 -shaft; 6, 7 - sealing $O$-rings; $\boldsymbol{8}$-casing; 9,10 - self-adjusting rings; 11 - rolling element; 12 - magnetic fluid

Fig. 7 shows the magnetic fluid seal for a shaft with large axial runout. In this construction two MF seals, consisting of magnets 7 , pole shoes 8 and the magnetic fluid 9 , are mounted in the carrying sleeves 5 . The flange 3 is mounted between the casing elements 1,2 .

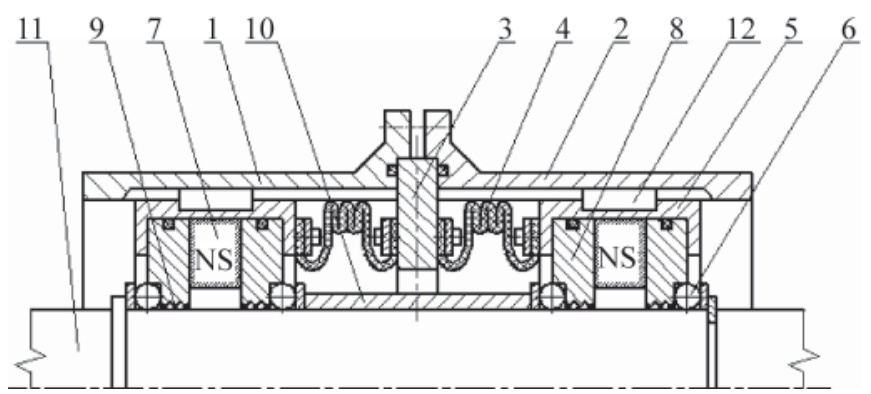

Fig. 7. Magnetic fluid seal for a shaft with large axial runout: 1, 2 -casing elements; 3 -flange; $\mathbf{4}$ - elastic bellows; $\mathbf{5}$-carrying sleeve $\mathbf{6}$-rolling element; 7 -permanent magnet; $\boldsymbol{8}$-pole shoe; $\mathbf{9}$-magnetic fluid; 10 -distance sleeve; 11 - shaft; 12 - key

The carrying sleeves 5 are connected with the flange 3 via elastic bellows 4 . The sleeves 5 can move in axial direction along the casing elements 1,2 . The rolling elements 6 allow the shaft 11 to rotate at constant dimensions of the working gaps between the lips of the pole shoes 8 and the shaft, with 
the magnetic fluid 9 filling these gaps. Axial dislocations of the shaft 11 are totally compensated by the deformations of the bellows 4 .

Fig. 8 shows a construction of the magnetic fluid seal for a vertical shaft with large axial and radial runout. The seal consists of the casing 3 , a magnetic sleeve 2 , a radially polarised permanent magnet 6 , a pole shoe 9 and the magnetic fluid 8 . The magnet 6 and the pole shoe 9 are mounted in the recess in the casing 3 . The closed magnetic circuit is composed by the casing 3 , the magnet 3 , the pole shoe 9 , the sleeve 2 , and the magnetic fluid 8 . The working gap between the sealing lips of the casing 3 and the pole shoe 9, and the sleeve 2 fixed on the shaft 1 , is approximately equal to $0.5-0.6 \mathrm{~mm}$ and is filled with the magnetic fluid 8 , which creates sort of a liquid gland and secures tightness of the centre. Axial and radial runout of the shaft 1 is compensated by the elastic bellows 13 mounted to the casing 3 and the machine body.

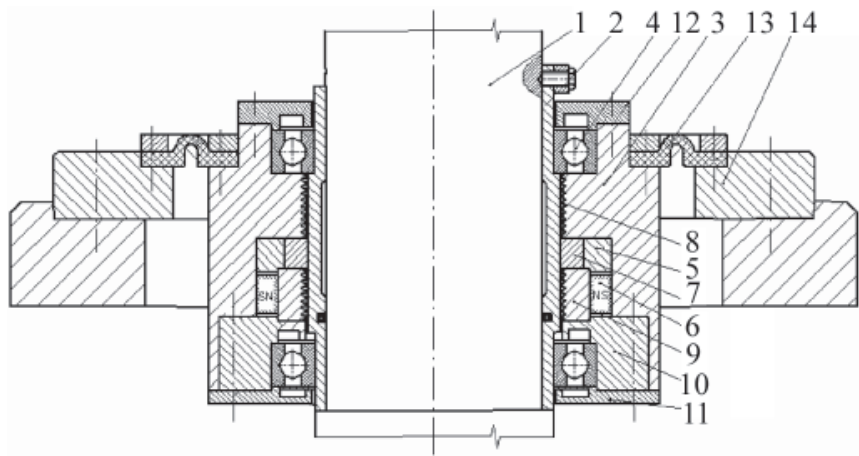

Fig. 8. Magnetic fluid seal for a vertical shaft with large axial and radial runout: $\mathbf{1}$-shaft; $\mathbf{2}$ - magnetic sleeve; $\mathbf{3}$-casing; $\mathbf{4}$-rolling bearing; $\mathbf{5}$ - non-magnetic ring; $\mathbf{6}$ - permanent magnet; 7 -felt ring; $\boldsymbol{8}$ - magnetic fluid; 9 -pole shoe; 10 - non-magnetic retaining ring; 11 -lower cover; 12 - upper cover; 13 - elastic bellows; 14 - flange

Fig. 9 shows other designs of magnetic fluid seals for shafts revealing large axial and radial runout [9]. In the seal shown in Fig. 9a the shaft 2 transmits the rotational motion with radial and axial runout to the sleeve 6 via an elastic element (bellows 7), which fully compensates those dislocations by own deformation. The elastic bellows 7 secures tight joint of the shaft 2 with the sleeve 6 . The rolling bearings 5 allow the sleeve 6 to rotate at constant working gap with respect to the fixed pole shoes 8 mounted in the casing 1, independently of radial and axial dislocations of the shaft 2 . The magnetic fluid 4 is situated in radial gaps between the lips of the pole shoes 8 and the outer surface of the sleeve 6 , and is kept inside by magnetic forces. In the design shown in Fig. 9b the MF seal is placed, along with the rolling bearings 5 , in the casing 1 and mounted on the shaft 2 . The casing 1 is connected via elastic bellows 7 with the stationary housing of the device. The above design also includes the system which cools the MF seal by delivering the cooling medium to the channels in the pole shoes.

\section{MAGNETIC FLUID SEALS USED IN HIGH-TEMPERATURE OPERATION CONDITIONS}

The lifetime of a magnetic fluid seal used in higher temperatures mainly depends on the temperature of intensive evaporation of the carrying liquid. The magnetic fluids used in these conditions are produced on the basis of specially silicone liquids or synthetic oils (Tab. 1, Tab. 2) which secure correct operation of the seal at temperatures up to $150^{\circ} \mathrm{C}$, with the critical temperature approximately equal to $200^{\circ} \mathrm{C}$.

Intensive heating of the magnetic fluid takes place in seals which operate in high temperature environment, and when

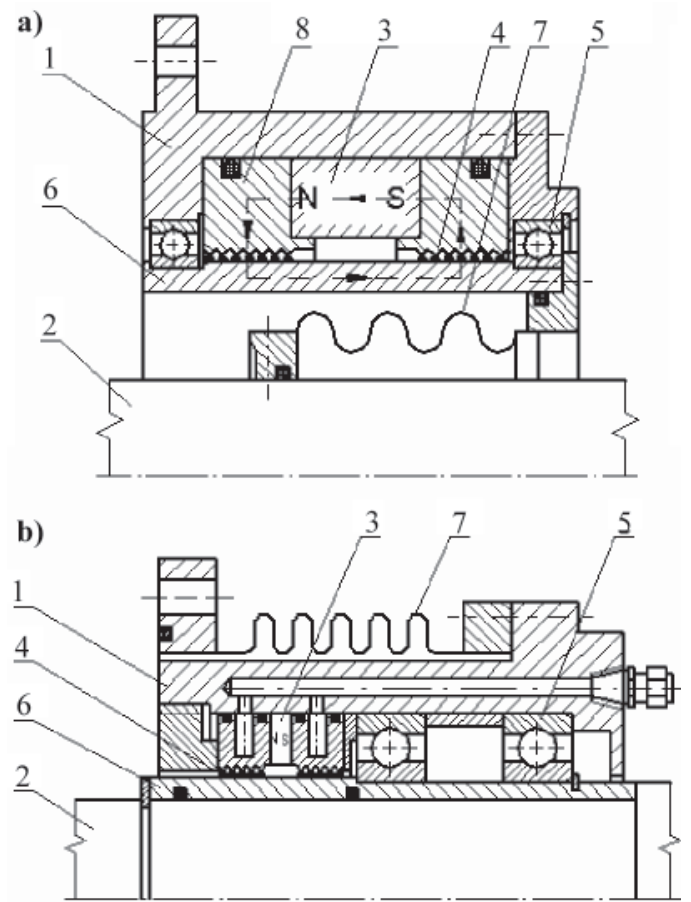

Fig. 9. Magnetic fluid seal for a shaft with large axial and radial runout: a), b) - design variants: 1 - casing, 2 - shaft, 3 - permanent magnet, 4 - magnetic fluid, 5 - rolling bearing, 6 -sleeve, 7 - elastic bellows, $\mathbf{8}(\boldsymbol{a})$ - pole shoe

they seal shafts with extremely high rotational speed, where the produced heat is provoked by internal friction in the fluid. In those cases seals with extra cooling systems are used.

Fig. 10 shows a multi-lip sealing culvert with magnetic fluid, produced by FerroLabs (Russia) [7], which is used in industrial high-temperature bioreactors. The culvert is mounted on the shaft 1 using a rotating sleeve 2 linked with the shaft via the clamping ring 6 . The sealing magnetic system is composed of the permanent magnet 7 and the pole shoes 8 mounted in the stationary sleeve 3 , the lips of the rotating sleeve 2 , and the magnetic fluid 9 in the gaps between the lips and the pole shoes. The geometry of the gap filled with the magnetic fluid is kept constant by the rolling bearings 4 and 5 .

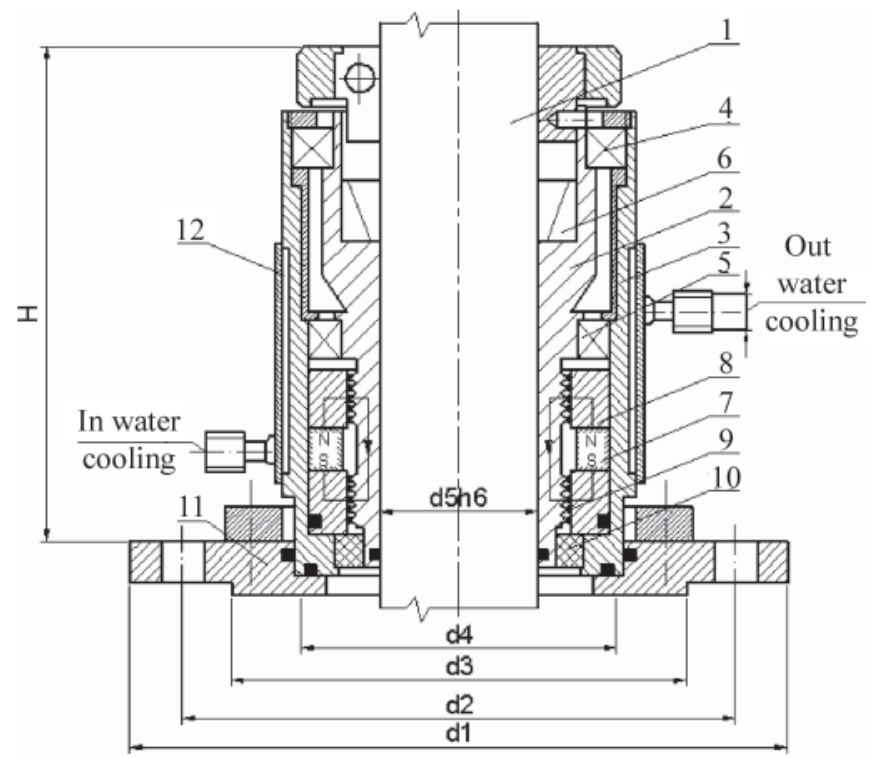

Fig. 10. Magnetic fluid seal for vertical shaft with external cooling system, made by FerroLabs: $\mathbf{1}$ - shaft; $\mathbf{2}$ - rotating sleeve; $\mathbf{3}$ - stationary sleeve; 4,5 - rolling bearings; $\boldsymbol{6}$ - clamping ring; 7 -permanent magnet; 8 -pole shoe; 9 - magnetic fluid; 10 - sliding ring; 11 -flange; 12 - water jacket 
A large part of length of the stationary sleeve 3 is surrounded by the water jacket 12 which protects the seal against the action of the ambient temperature. At the same time the water carries away the heat generated in the magnetic liquid during highspeed operation of the shaft.

According to the catalogue data given by the producer, this seal can work at ambient temperatures up to $1200^{\circ} \mathrm{C}$ and at maximum peripheral speed $15 \mathrm{~m} / \mathrm{s}$, when the pressure does not exceed 0.3 $\mathrm{MPa}$. This culvert, produced for shafts with diameters ranging from 40 to $100 \mathrm{~mm}$, can also work at the presence of lateral vibrations of the shaft, the amplitude of which does not exceed do $0.7 \mathrm{~mm}$.

Fig. 11 shows a magnetic fluid seal designed for highspeed shaft with external and internal cooling system [9]. In this design the cooling liquid (water) is delivered to the seal by channels 9 made in the pole shoes 5, 6 and in the hollow shaft 2 .

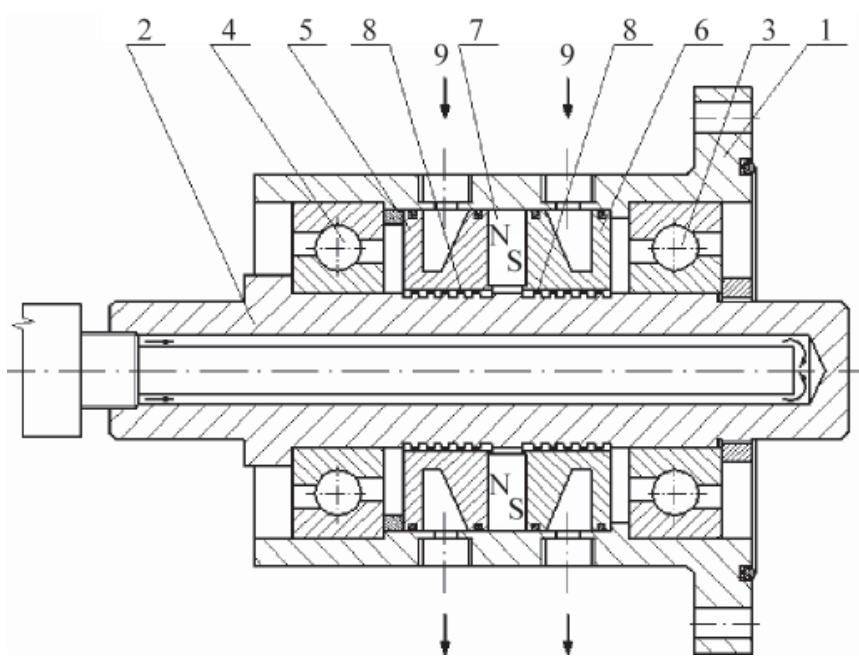

Fig. 11. Magnetic fluid seal for high-revolution shaft with external and internal cooling system: 1 - casing; 2 - hollow shaft; 3, 4-rolling bearing; $\mathbf{5}, \mathbf{6}$-pole shoes; 7 - permanent magnet; 8 - magnetic fluid; 9 -channel

\section{MAGNETIC FLUID SEALS USED IN LIQUID ENVIRONMENT}

Magnetic fluid seals which are used for sealing rotating shafts in liquid environment require double sealing systems, in which the MF seal is used as the second seal following a conventional seal in a one-by-one arrangement. This solution limits direct contact of the sealed liquid with the magnetic fluid, and the entire sealing construction secures absolute tightness of the system.

Fig. 12 presents a hybrid seal for an impeller pump shaft. This seal consists of a centrifugal seal and a magnetic fluid seal [10]. The first sealing stage is the centrifugal seal 4 having the form of a disc mounted on the shaft 1 . The second sealing stage is the magnetic fluid seal, consisting of the permanent magnet 5 , the pole shoe 6 and the magnetic fluid 7. On the shaft 1 made of material revealing good magnetic permeability, two sealing lips $1 \mathrm{a}$ are mounted under the pole shoe 6 . The magnetic fluid 7 fills small ring gaps between the pole shoe 6 and the sealing lips $1 \mathrm{a}$, and is kept in there by magnetic forces to create additional sealing obstacle for the working medium. The chamber 8 filled with the compressed neutral gas protects the sealed medium (water, for instance) against coming into direct contact with the magnetic fluid, eliminating this way an unfavourable effect of the working medium on the magnetic fluid.

Fig. 13 presents a hybrid magnetic fluid seal for a vertical shaft of a deep-well pump [11]. This seal is a composition of a centrifugal seal with two MF seals, out of which one MF seal is situated above the centrifugal seal and the other - below. The centrifugal seal consists of a rotor, having the form of the flange 9 mounted on the shaft 1 situated in the chamber 11, and the barrier fluid 10. In dynamic conditions the sealing function is performed by the centrifugal seal while in static conditions - by two MF seals, which counteract the leakage of the barrier fluid from the chamber and the penetration of impurities to pump inside.

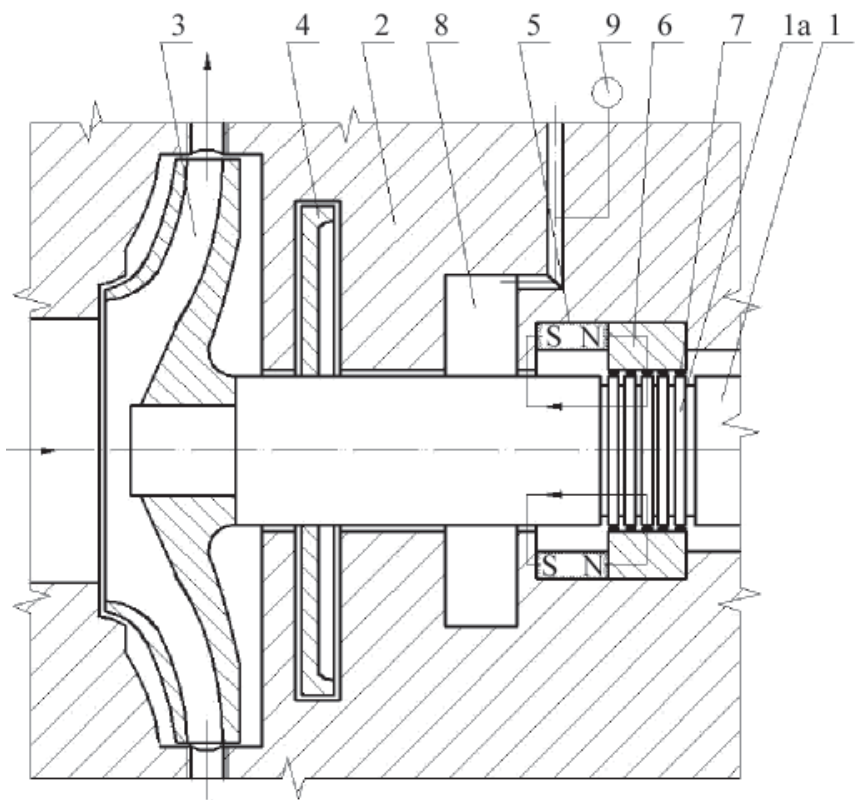

Fig. 12. Hybrid seal of impeller pump shaft: 1 - shaft, $1 \boldsymbol{a}$ - sealing lip $\mathbf{2}$ - casing, $\mathbf{3}$-impeller, 4 - centrifugal seal, $\mathbf{5}$ - permanent magnet, $\mathbf{6}$ - pole shoe, 7 -magnetic fluid, $\mathbf{8}$-chamber filled with neutral gas, 9 - manometer

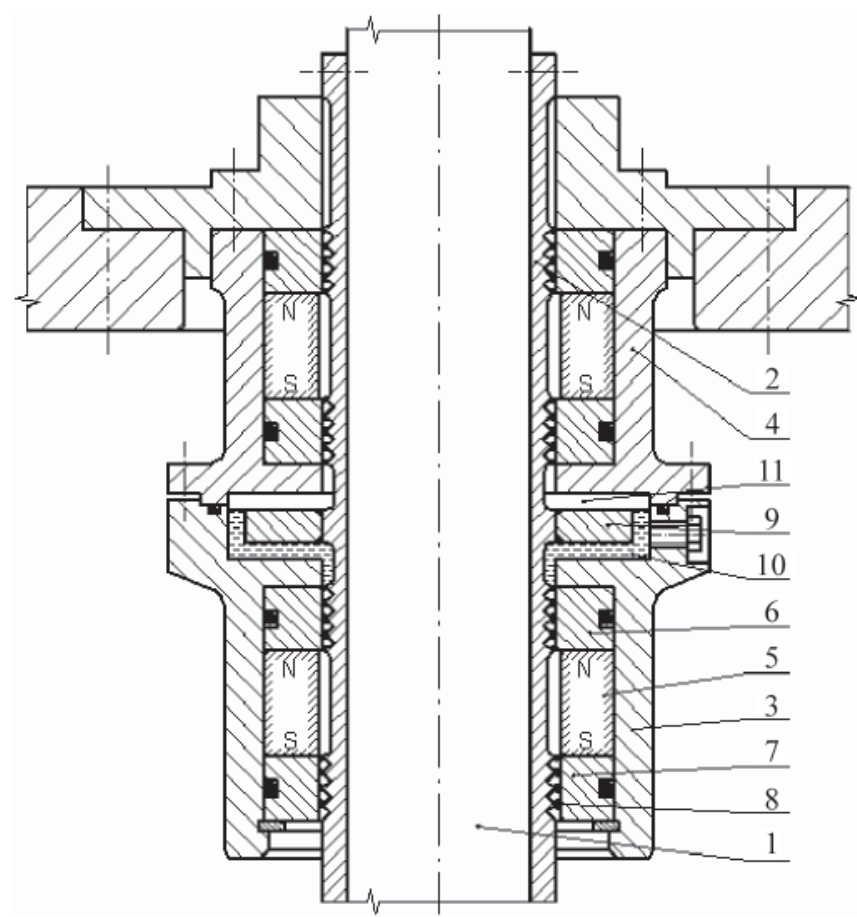

Fig. 13. Hybrid magnetic fluid seal for a vertical pump shaft 1 -shaft; 2 -sleeve with sealing lips; 3, 4-casing elements, $\mathbf{5}$ - permanent magnet; 6,7 -pole shoes; 8 - magnetic fluid; 9 - flange; 10 - barrier fluid; 11 - chamber

Fig. 14 presents the double rotating shaft seal consisting of the primary liquid seal, the screw seal (Fig.14a) or the centrifugal seal (Fig.14b) for instance, and the secondary magnetic fluid seal situated behind the primary seal [9]. In this case the basic sealing function in dynamic conditions is 
performed by the conventional liquid, while the role of the MF seal is to keep the working medium inside the machine in steady-state conditions and at low shaft rotations.

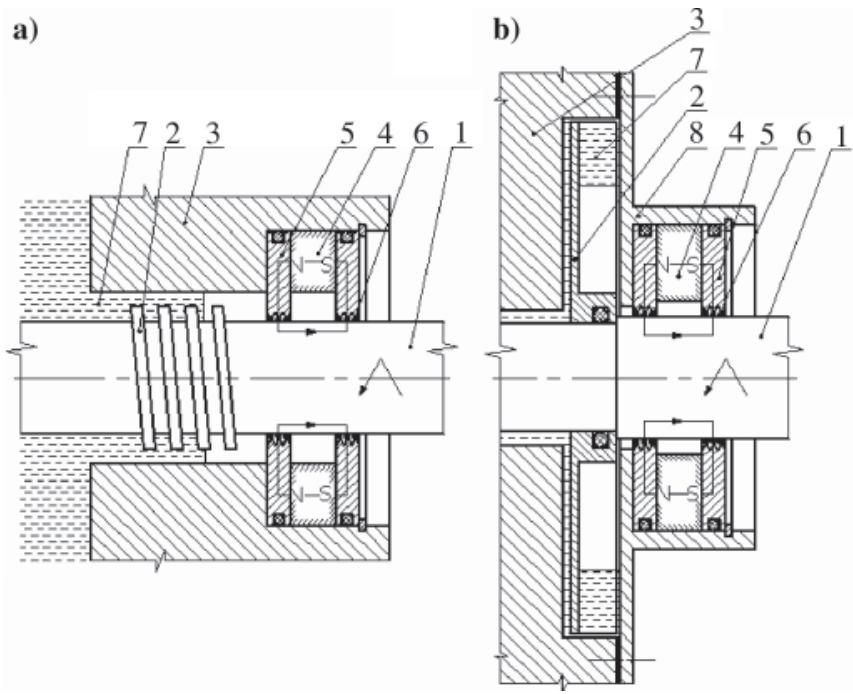

Fig. 14. Double rotating shaft seal: a) composed of screw seal and magnetic fluid seal; b) composed of centrifugal seal and magnetic fluid seal: 1 - shaft, 2 -screw groove or rotor, 3 - casing, 4 -permanent magnet, 5 - pole shoe, $\mathbf{6}$ - magnetic fluid, 7 - sealed liquid, $8(\boldsymbol{b})$ - cover

Fig. 15a presents a general view of a sea-going vessel with marked the position of the propeller drive system, while in Fig. $15 \mathrm{~b}$ the drive system itself is schematically shown with marked the position of the passage that seals the propeller shaft. Fig. 15c shows a typical packing seal with soft packing, normally used for ship propeller shafts. This seal consists of a package of sealing rings 6 made of a woven cord which are placed in the packing chamber and pressed by the gland 5 . A disadvantage of this solution is the need for periodical pressing of the gland to compensate the relaxation of stresses in the packing.

Fig. 15d shows the construction of a magnetic fluid seal for the propeller shaft, which protects against the penetration of the seawater inside the ship's hull [12]. This seal consists of two axially polarised permanent magnets 6,7 , separated by multiedge pole shoes 8,9 and the magnetic fluid 10. The magnets 6, 7 and the pole shoes are mounted in the non-magnetic sleeve 5 fixed in the seal casing 4 . Two closed magnetic circuits are composed by the magnets 6,7 , the pole shoes 8,9 , the magnetic fluid 10 and the shaft 1 made of material revealing good magnetic permeability. The magnetic fluid 10 is kept by the magnetic forces inside small ring gaps between the sealing lips of the pole shoes 8,9 and the shaft 1 , thus creating the sealing barriers for the working medium.

Fig. 16 shows the construction of a two-stage seal for a ship propeller shaft. The seal consists of a $n$ umber of lip seals and the magnetic fluid seal [13]. The first sealing stage consists of two sealing lip rings 4 mounted in the casing 2 . The second stage is the magnetic fluid seal which consists of the axially polarised permanent magnet 5 , two pole shoes 6 and the magnetic fluid 7. The closed magnetic circuit is composed by the magnet 4 , the pole shoes 6 , the magnetic fluid 7 and the sleeve 3 , made of material revealing good magnetic permeability, which is mounted on the shaft 1 . The magnetic forces keep the magnetic fluid inside the small ring gaps 8, thus composing additional barriers for the sealed medium.

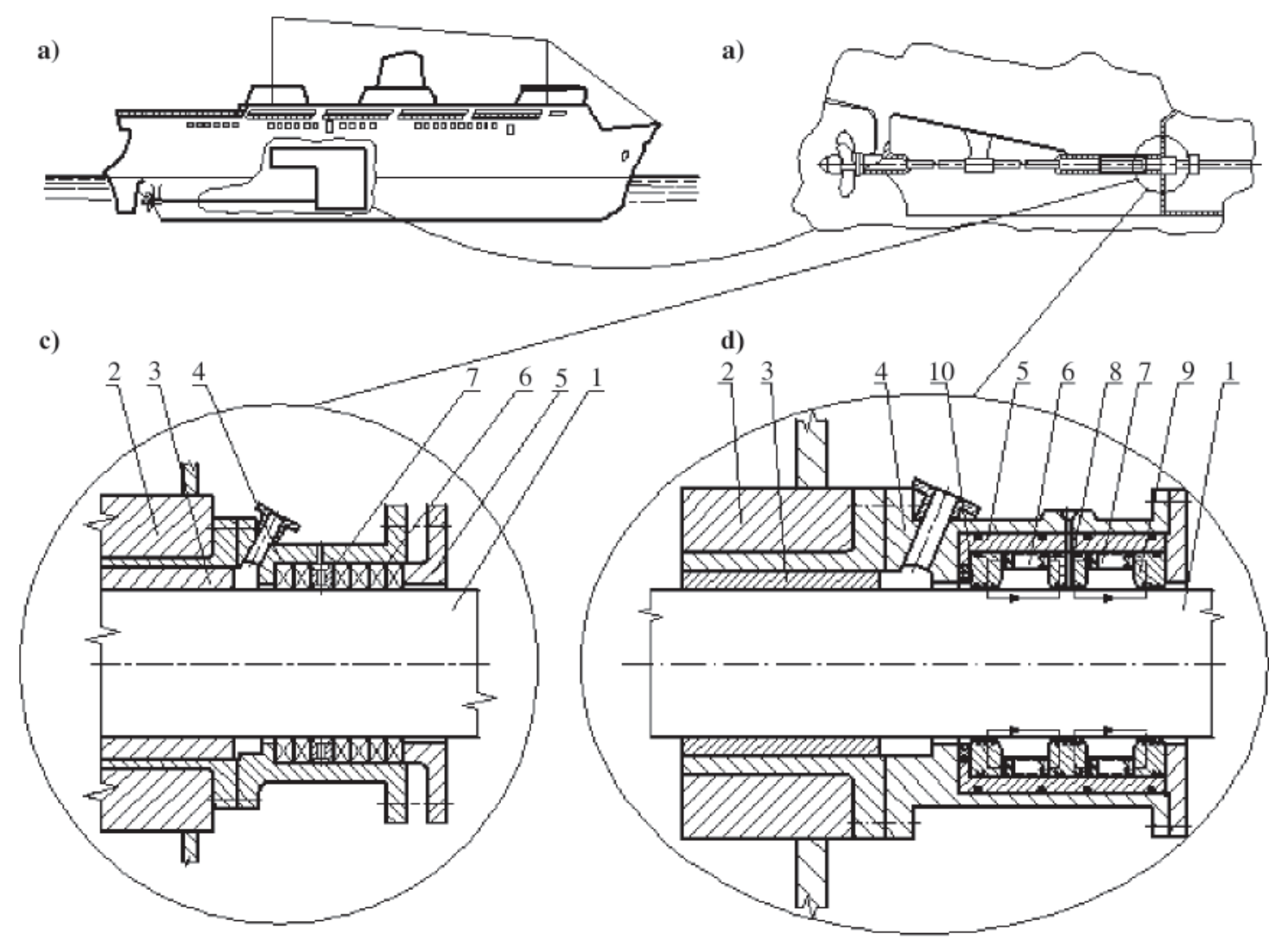

Fig. 15. Seal for ship propeller shaft: $\boldsymbol{a})$ sea-going vessel with marked position of the propeller drive system; $\boldsymbol{b})$ scheme of propeller drive system; c) typical packing seal for propeller shaft: $\mathbf{1}$ - propeller shaft, $\mathbf{2}$ - body, $\mathbf{3}$ - slide bearing, $\mathbf{4}$-seal casing, 5 - gland, 6 - package of sealing rings, 7 -distance ring; d) magnetic fluid seal: $\mathbf{1}$ - propeller shaft; 2 - body; 3 - slide bearing; 4 -seal casing; 5 - non-magnetic sleeve; 6, 7 -permanent magnets; 8,9 -multi-edge pole shoes; 10 - magnetic fluid 


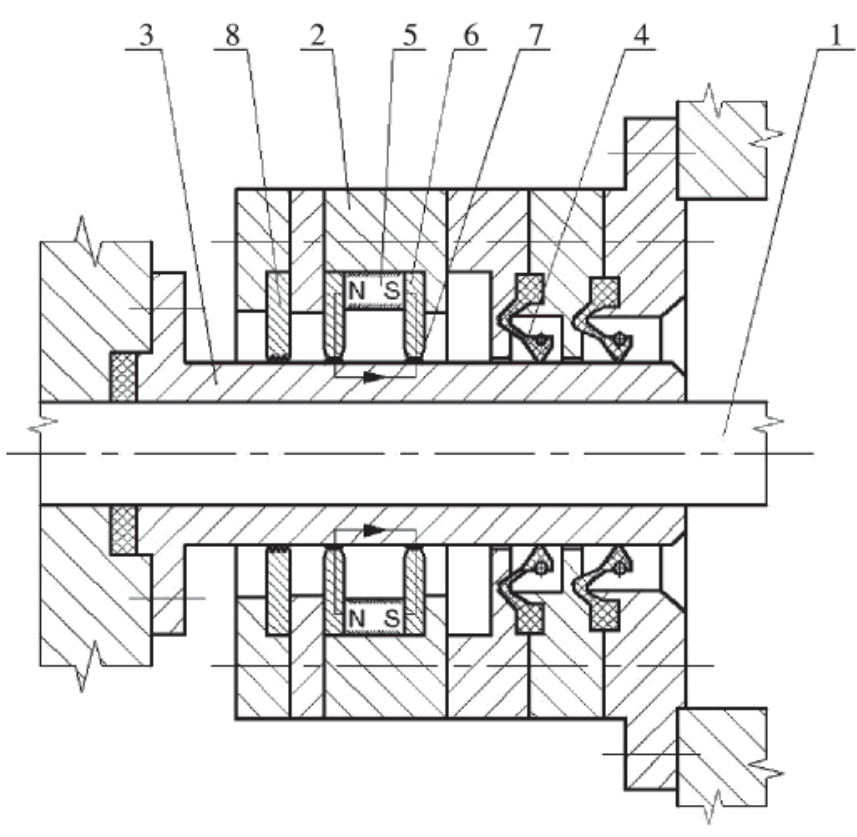

Fig. 16. Two-stage seal for ship propeller shaft, composed of lip seals and magnetic fluid seal: $\mathbf{1}$ - ship propeller shaft, $\mathbf{2}$ - casing, $\mathbf{3}$ - protecting sleeve, 4 -sealing lip ring, $\mathbf{5}$-permanent magnet, $\mathbf{6}$-pole shoe, 7 - magnetic fluid, $\boldsymbol{8}$ - sealing groove/gap ring

\section{RESEARCH ACTIVITIES CARRIED OUT IN THE AGH LABORATORY OF SEALS AND MAGNETIC FLUID APPLICATIONS OVER THE USE OF MAGNETIC FLUIDS FOR SEALING ROTATING SHAFTS IN WATER ENVIRONMENT}

Research and design activities are carried out in the AGH Laboratory of Seals and Magnetic Fluid Applications over the use of magnetic fluid seals in water environment. These activities are oriented on working out MF seals able to work effectively in machines in which low-pressure water flow takes place.

The objects of experimental investigations are seals having two different nominal diameters: $50 \mathrm{~mm}$ and $220 \mathrm{~mm}$. This situation forced building two research rigs of different construction.

The experimental investigations of the seal of $50 \mathrm{~mm}$ in diameter, performed in water at pressure equal to $0.1 \mathrm{MPa}$ and rotational speed equal to $3000 \mathrm{rev} / \mathrm{min}$, have proved that this seal can continuously work during 200 hours, the least, without any traces of worsening of the assumed initial parameters. Tests performed on different constructional versions of this seal have revealed potential for increasing the critical speed of the shaft by using additional protecting elements in the sealing unit.

For the investigations of the magnetic fluid seal of $220 \mathrm{~mm}$ in nominal diameter, a special research rig was built which made it possible to measure the torque on the propeller shaft, the temperature of the seal, and its tightness in the presence, or absence, of the flow of the process liquid (water, in the examined case) through the sealed research chamber.

Fig. 17 shows a cross-section of the research head used in this rig. The basic elements of this head include the collapsible chamber 1,2, 3, 4, with a set of magnets 7 with pole shoes 5 . In the chamber the rotating disc 13 is placed, on which the rim 14 with sealing lips is mounted. The disc is fixed on the shaft 9 using the clamping ring 16.

After the magnetic fluid is introduced to the gaps between the pole shoes 5 and the sealing lips of the rotating disc 13, the chamber is divided into space A filled with the examined medium, and space B used for disposing of leakages through the opening E drilled in the lower part of the chamber.

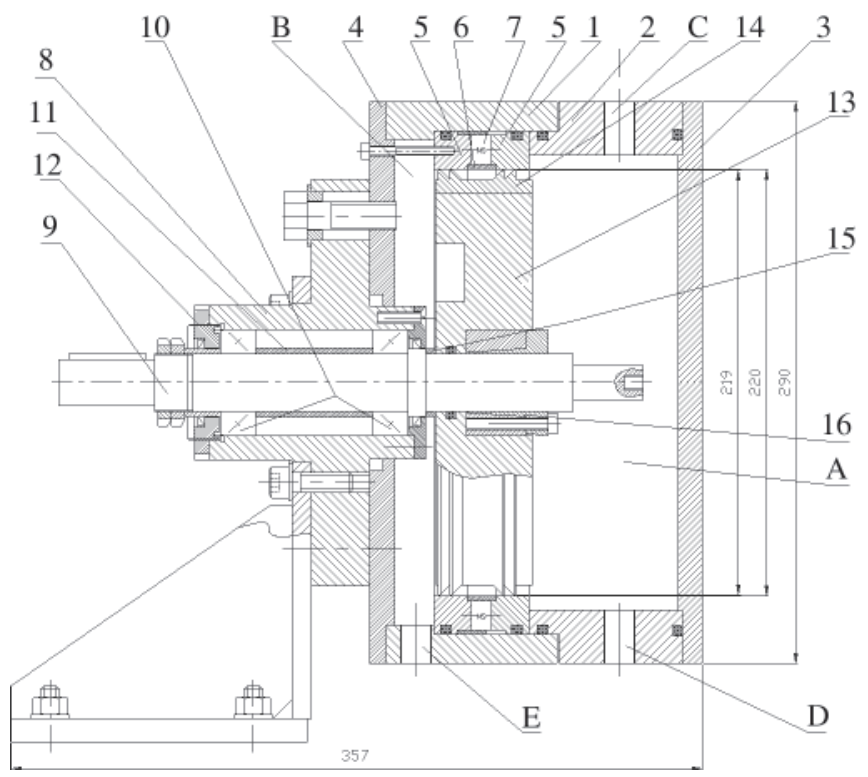

Fig. 17. Research head used on the rig for examining large-dimension magnetic fluid seals in water environment-MFLP1: 1, 2, 3, 4-components of the collapsible research chamber; 5 -pole shoes; $\mathbf{6}$ - non-magnetic distance sleeve; 7 - replaceable set of permanent magnets $7 ; \boldsymbol{8}$-shaft body; 9 -rotating shaft; 10 - cone bearings; 11 -distance sleeve; 12 -adjusting nut; 13, 14 - two-part disc with sealing lips; 15 - positioning sleeve; 16 - clamping sleeve.

The openings $\mathrm{C}$ and $\mathrm{D}$ in the chamber are used for filling the space A, or for generating the flow of the examined medium with the aid of an external hydraulic system.

A basic condition for correct action of the magnetic fluid seal is securing uniform thickness of the gap along the entire perimeter of the seal.

Meeting this condition is difficult on the research rig designed for examining large-diameter objects, as the examination of different geometric versions of the seal would require a rig construction in which particular elements could be changed, at the same time securing the repeatability of the obtained results.

The research rig under discussion was tested in conditions in which the sealed chamber was filled with gas (air) and with liquid (water) under pressure $0,05 \mathrm{MPa}$ and without flow.

The performed investigations and geometrical measurements have revealed high influence of the magnetic field generated by the set of permanent magnets on the axial alignment of the rotating and fixed elements of the seal when the magnetic circuit was closed by the magnetic fluid. This phenomenon was a source of leakages through the sealing magnetic fluid, and resultant difficulties in keeping the required pressure in the research chamber, which was particularly visible at low rotational speed ranges.

That was why additional elements were to be machined and installed in the rig to centre the set of pole shoes with respect to the rotating disc with sealing lips.

The measurements and investigations performed in the presence of additional centring elements have confirmed high efficiency of the introduced constructional changes.

The investigations did not take long, as their main goal was to check the operation of the research rig. Half-hour tests were performed with the water under pressure $0.03 \mathrm{MPa}$ in the chamber and at shaft rotational speeds equal to $250 \mathrm{rev} / \mathrm{min}$, $500 \mathrm{rev} / \mathrm{min}$ and $600 \mathrm{rev} / \mathrm{min}$.

The modernised construction has confirmed the ability of the magnetic fluid seal with the working gap having 
the nominal thickness of $0.5 \mathrm{~mm}$ to keep pressure in the abovementioned time interval and at the abovementioned working conditions.

\section{BIBLIOGRAPHY}

1. Berkovsky B.M., Medvedev V.F., Krakov M.S.: Magnetic fluids - engineering applications, Oxford Science Publications, 1993

2. Rosensweig R.E.: Ferrohydrodynamics, Cambridge University Press, Cambridge, MA, 1985

3. Orlov D.W., Podgorkov W.S.: Magnitnyje żidkosti w maszinostrojenii, Izd. Maszinostrojenije, Moskwa, 1993

4. Kamiyama S., Oyama T., Htwe J. : Basic study on the performance of magnetic fluid seals, pp. 985-990. Proc. of JSLE Int Tribology Conf. 1985

5. J. Kurfess, H.K. Müller : Sealing liquids with magnetic fluids, Journal of Magnetism and Magnetic Materials, 85, 1990

6. Lawniczak A., Milecki A.: Electro- and magnetorheological fluids and their technical applications, Publishing House of Poznan University of Technology, Poznan, 1999 (in Polish)

7. Catalogue: Ferrofluid-based seals, Ferrolabs Inc. (Rosja)

8. Product Bulletin: Magneto-Rheological Fluids, LORD Corp. (USA)

9. Tietze W.: Handbuch Dichtungspraxis, 3.Auflage, VulkanVerlag, Essen 2003
10.USA patent, Int.Cl. F16J15/40, no 4681328, Magnetic fluid shaft seal

11.USA patent, Int.Cl. F 16J15/42, no 4054293, Hybrid magnetic fluid shaft seal

12.USA patent, Int.Cl. F16J15/54, no 4436313, Device for sealing a propeller shaft against invasion of sea water

13.JP patent, Int.Cl. F16J15/16, no 62178498, Stern pipe sealing device

\section{CONTACT WITH THE AUTHORS}

Leszek Matuszewski, Ph. D.

Faculty of Ocean Engineering and Ship Technology

Gdansk University of Technology

Narutowicza 11/12

80-952 Gdansk, POLAND

e-mail : leszekma@pg.gda.pl

Zbigniew Szydło, Ph.D.

AGH University of Science and Technology Faculty of Mechanical Engineering and Robotics Al. Mickiewicza 30

31-069 Kraków, POLAND zbszydlo@agh.edu.pl

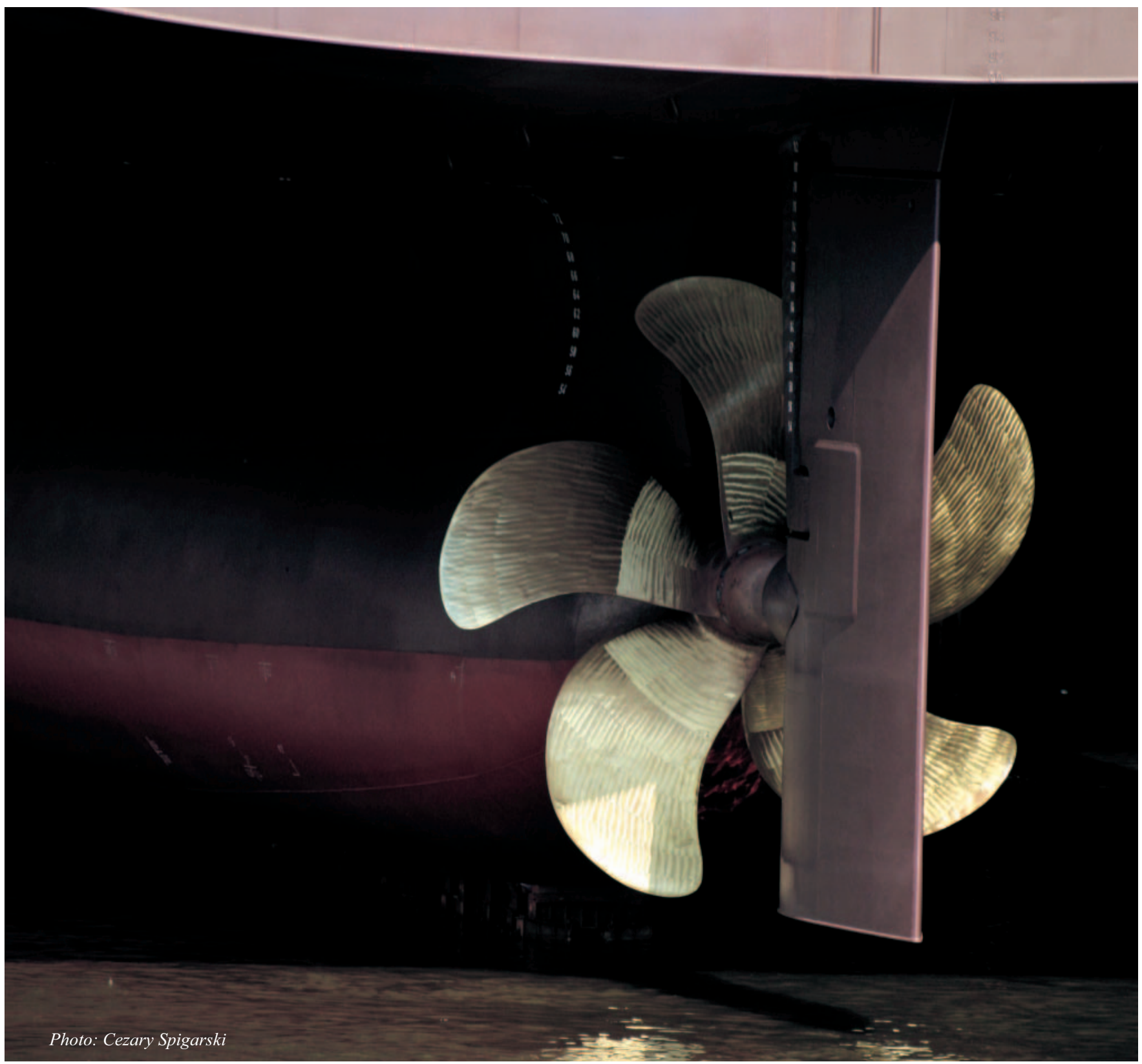

